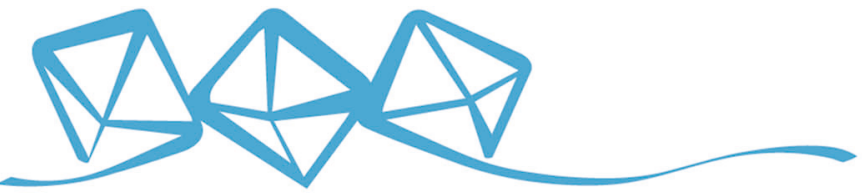 COMMUNICATIONS MATERIALS
}

ARTICLE

Check for updates https://doi.org/10.1038/s43246-020-0040-3 OPEN

\section{Imaging switchable magnetoelectric quadrupole domains via nonreciprocal linear dichroism}

\author{
Kenta Kimura $^{1 凶}$, Tsukasa Katsuyoshi ${ }^{1}$, Yuya Sawada ${ }^{2}$, Shojiro Kimura ${ }^{2}$ \& Tsuyoshi Kimura (1) ${ }^{1}$
}

Parity-odd magnetoelectric multipoles such as magnetic quadrupoles and toroidal dipoles contribute to various symmetry-dependent magnetic phenomena and formation of exotic ordered phases. However, the observation of domain structures emerging due to symmetry breaking caused by these multipoles is a severe challenge because of their antiferromagnetic nature without net magnetization. Here, we report the discovery of nonreciprocal linear dichroism for visible light $(\sim 4 \%$ at $1.8 \mathrm{eV})$ in a magnetic quadrupole ordered phase of antiferromagnetic $\mathrm{Pb}(\mathrm{TiO}) \mathrm{Cu}_{4}\left(\mathrm{PO}_{4}\right)_{4}$, which enables the identification of magnetic quadrupole domains of opposite signs. Symmetry considerations indicate that nonreciprocal linear dichroism is induced by the optical magnetoelectric effect, i.e., the linear magnetoelectric effect for electromagnetic waves. Using the nonreciprocal linear dichroism, we successfully visualize spatial distributions of quadrupole domains and their isothermal electric-field switching by means of a transmission-type polarized light microscope. The present work exemplifies that the optical magnetoelectric effect efficiently visualizes magnetoelectric multipole domains responding to external perturbations.

\footnotetext{
${ }^{1}$ Department of Advanced Materials Science, University of Tokyo, Kashiwa 277-8561, Japan. ${ }^{2}$ Institute for Materials Research, Tohoku University, Sendai 980-8577, Japan. ${ }^{{ }}$email: kentakimura@edu.k.u-tokyo.ac.jp
} 
$\mathrm{M}$ agnetic symmetry of matter plays an essential role in defining their physical properties associated with magnetism, such as magnetoelectric, magnetooptical, and magnetotransport properties. Recent theoretical studies have shown that such intriguing magnetic phenomena are fully described by $n$-th rank magnetic multipoles and magnetic toroidal multipoles from the symmetry viewpoint ${ }^{1,2}$. Among them, magnetic multipoles with rank 0 (monopole) and rank 2 (quadrupole), and magnetic toroidal multipole with rank 1 (toroidal dipole) are categorized as the most fundamental parity-odd magnetic quantities. They are referred to as magnetoelectric (ME) multipoles ${ }^{3}$, because their ferroic ordering breaks both macroscopic time-reversal and space-inversion symmetries and therefore can exhibit a linear ME effect, $\mathbf{P}=\chi \mathbf{B}$ and $\mathbf{M}=\chi^{\mathrm{tr}} \mathbf{E}$, namely, a linear induction of electric polarization $\mathbf{P}$ (magnetization $\mathbf{M}$ ) by a magnetic field $\mathbf{B}$ (an electric field $\mathbf{E})^{4-7}$. The coupling is defined by the second rank ME tensor $\chi$ that has nine independent matrix elements $\chi_{i j}(i, j=a, b, c)$ corresponding to nine kinds of the ME multipole components ${ }^{7,8}$. The importance of the ME multipoles is exemplified in various magnetic systems. For example, since the ME multipoles are intrinsically antiferromagnetic entities, they play a key role for emergent ME coupling phenomena in antiferromagnets $7,9,10$. Also, they are carried by a spin texture such as (anti)skyrmions ${ }^{11}$, thus having a close relationship to functional properties of such topological magnetic phases. Moreover they are discussed in relation to unconventional superconductivity, e.g., in high- $T_{c}$ cuprates ${ }^{12,13}$.

In the study of functional properties and exotic orders associated with the ME multipole ordering, however, there is a crucial but challenging task. This is the observation of their domains, i.e., spatial distribution of a pair of ME multipoles of opposite signs that are spontaneously formed upon ferroic ordering. The difficulty mainly arises from the antiferromagnetic nature of the $\mathrm{ME}$ multipoles. The resulting absence of macroscopic magnetization causes an insensitivity to the well-known linear magneto-optical effect, which is commonly utilized to observe ferromagnetic domains. Nonlinear optics is known as an efficient tool to observe various types of antiferromagnetic domains including ME multipole domains ${ }^{14}$. However, it requires an intense pulsed laser and a relatively long exposure time (typically several minutes).

The emergence of the linear optical magnetoelectric (OME) effect, the linear ME effect for electromagnetic waves, is one of the most prominent signatures of ME multipoles ${ }^{15-19}$. They manifest as the unconventional nonreciprocal optical responses and have been observed in various wavelength regions ranging from microwave, terahertz, visible light, to $\mathrm{X}$ ray $^{20-29}$. Concerning the light propagation in a linear ME medium, the oscillating magnetic field $\left(\mathbf{B}^{\omega}\right)$ of the light magnetoelectrically induces an oscillating electric polarization $\mathbf{P}_{\mathrm{ME}}^{\omega}=\chi^{\omega} \mathbf{B}^{\omega}\left(\propto \chi^{\omega} \mathbf{k} \times \mathbf{E}^{\omega}\right)$, which changes the optical constants of the medium. Here, $\chi^{\omega}$ denotes $\chi$ at a finite frequency $\omega$ and $\mathbf{k}$ is the propagation vector of the light ${ }^{16,17}$. The sign of $\mathbf{P}_{\mathrm{ME}}^{\omega}$ is reversed upon either reversal of the light propagation direction or switching of the $\chi^{\omega}$ sign. The former feature leads to directionaldependent nonreciprocal optical responses such as gyrotropic birefringence ${ }^{20}$ and directional dichroism (optical diode effect) ${ }^{24-28}$. On the other hand, the latter feature makes it possible to discern two distinct magnetic domains that have ME tensors of opposite signs ${ }^{15,29}$. These features in the case of the magnetic quadrupole system are schematically illustrated in Fig. 1a-d. Such domaindependent optical responses open up the possibility of optical microscopy imaging of domain structures of ME multipoles (see Fig. 1e). Though such an imaging possibility was originally proposed in the $1960 \mathrm{~s}^{15}$, no experimental demonstration has been reported to date. This is most likely because the magnitude of the OME effect in AFM materials has never exceeded $10^{-4}$ for near-infrared to visible light regions ${ }^{20-22}$, which is too small for imaging application.
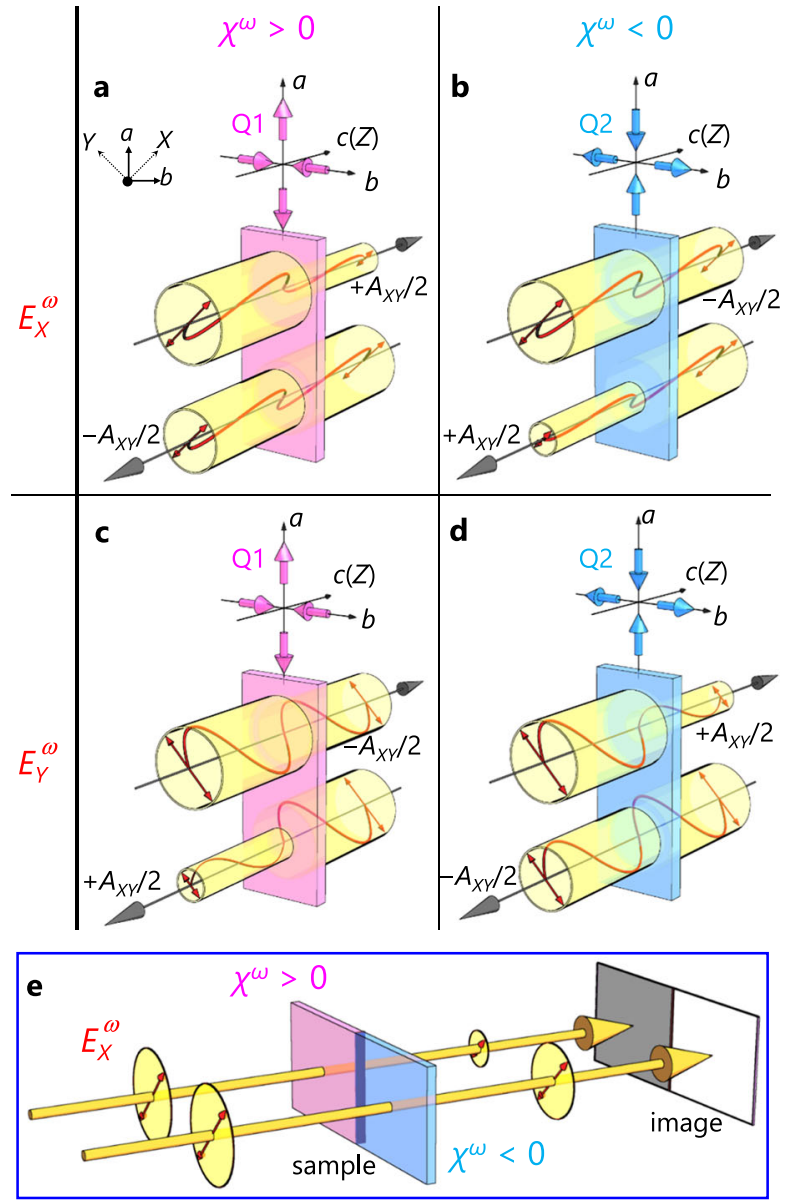

Fig. 1 Nonreciprocal light absorption induced by optical magnetoelectric (OME) effects. Optical absorption of the $a b-(X Y-)$ plane sample in various conditions in terms of the sign of the ME coefficient $( \pm \chi)$, light propagation direction $(\mathbf{k} \| \pm Z)$, and the polarization direction of the light $\left(\mathbf{E}^{\omega}\right)$. The length and orientation of the red double-headed arrows denote the intensity and the direction of $\mathbf{E}^{\omega}$. The OME contribution to the absorption coefficient $A$ is reversed between light with $+k_{z}$ and $-k_{z}$ (i.e., nonreciprocity) and between multipole domains with $\chi^{\omega}$ of opposite signs, as shown in $\mathbf{a}$ and $\mathbf{b}$. The latter feature of the OME effects allows for the domain imaging of the ME multipole order by means of the optical microscopy, as illustrated in e. In particular case of the ME quadrupole $\pm a_{a^{2}-b^{2}}$ with $\pm \chi_{X Y}^{\omega}= \pm \chi_{Y X X}^{\omega}$, whose spin arrangement is represented by closed pink or light blue arrows, the OME contribution is further reversed by the light polarization switching between $E_{X}^{\omega}$ and $E_{Y}^{\omega}$, as shown in $\mathbf{a}$ and $\mathbf{c}[\mathbf{b}$ and $\mathbf{d}]$. This phenomenon is called nonreciprocal linear dichroism. In this case, the OME contribution to $A$ in all the panels is described only by the linear dichroism $\Delta A_{X Y} \equiv \Delta A_{X}-$ $\Delta A_{Y}$.

Here, we report on the observation of a large OME effect for visible light in a tetragonal antiferromagnet $\mathrm{Pb}(\mathrm{TiO}) \mathrm{Cu}_{4}\left(\mathrm{PO}_{4}\right)_{4}$ (hereafter, abbreviated as $\mathrm{PbTCPO}$ ), whose magnetic structure is viewed as a ferroic ME quadrupole order ${ }^{30}$. Remarkably, light propagating along the tetragonal axis exhibits a large nonreciprocal linear dichroism up to $4 \%$, which is attributable to the strong light-polarization-dependent OME effect of pure quadrupole origin. Moreover, with such a large effect, we demonstrate optical imaging of quadrupole domains by using a transmission-type polarized light microscope with an exposure time of $0.5 \mathrm{~s}$ or shorter. By this technique, we also achieve the visualization of an isothermal electric-field switching of the quadrupole domains. 


\section{Results}

Magnetoelectric characteristics of target material. $\mathrm{PbTCPO}$ studied here is a blue transparent antiferromagnet crystallizing into a tetragonal structure with the space group $P 42_{1} 2$ (magnetic point group $4221^{\prime}$ ). In the following, the [110], [110], and [001] (c) axes are referred to as $X, Y$, and $Z$, respectively. The magnetic properties of $\mathrm{PbTCPO}$ are dominated by a staggered array of upward and downward $\mathrm{Cu}_{4} \mathrm{O}_{12}$ square cupola clusters in the $X Y$ plane (see Fig. 2a). An antiferromagnetic phase transition takes place at a temperature of $T_{\mathrm{N}}=7.0 \mathrm{~K}$, below which finite $\mathbf{P}$ develops in the presence of $\mathbf{B}$ (see Supplementary Fig. 1). No substantial net magnetization is present in the absence of $\mathbf{B}$ (see Supplementary Fig. 2). The magnetic structure indicated by neutron diffraction measurements $\mathrm{s}^{30}$ is shown in Fig. 2a. The magnetic point group is $4^{\prime} 22^{\prime}$, which maintains the tetragonal symmetry, but breaks both space-inversion and time-reversal

a

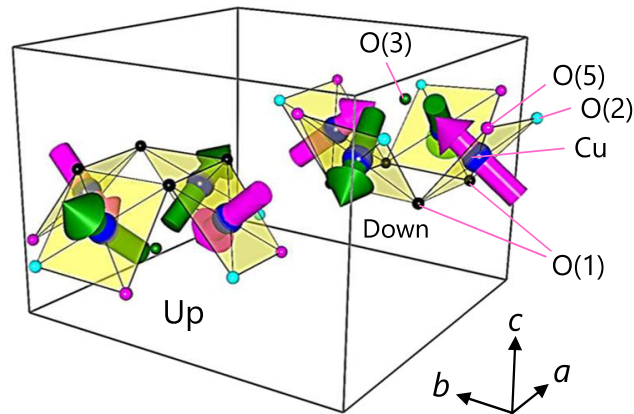

b

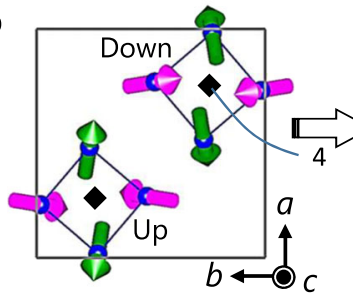

Q1 $\left(\chi_{a a}>0\right)$

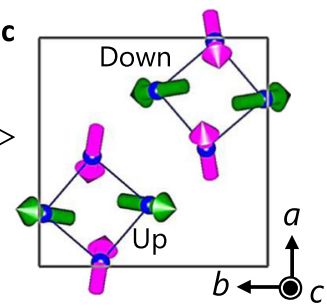

Q2 $\left(\chi_{a a}<0\right)$

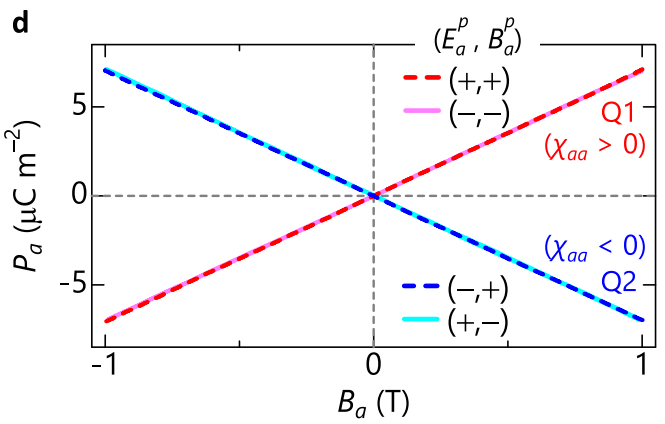

Fig. 2 Magnetic structure and domains in the antiferromagnetic phase of $\mathbf{P b}(\mathrm{TiO}) \mathrm{Cu}_{\mathbf{4}}\left(\mathbf{P O}_{\mathbf{4}}\right)_{\mathbf{4}}$. a A bird's eye view of the crystal and magnetic structures. $\mathrm{Pb}, \mathrm{Ti}$, and $\mathrm{P}$ atoms are omitted. $\mathrm{A}$ unit cell (solid line) contains one upward and one downward $\mathrm{Cu}_{4} \mathrm{O}_{12}$ square cupola each formed by four $\mathrm{Cu}^{2+}$ ions (large blue balls) and twelve $\mathrm{O}^{2-}$ ligands (small balls). Magenta and green arrows represent inward and outward pointing $\mathrm{Cu}^{2+}$ spins on the square cupolas, respectively. The $c$-axis views of the magnetic structures in b Q1 and c Q2 quadrupole domains, which are mutually converted by either fourfold-rotation (4) or time-reversal operations. The ME coefficient $\chi_{a a}$ in these two domains is opposite in sign to each other. $\mathbf{d}$ Electric polarization along the $a$ axis as a function of magnetic field along the $a$ axis at $3 \mathrm{~K}$, measured after cooling with four different sign combinations of poling electric $\left(E_{a}^{p}= \pm 0.83 \mathrm{MV} \mathrm{m}^{-1}\right.$ ) and magnetic fields along the $a$ axis $\left(B_{a}^{p}= \pm 0.1 \mathrm{~T}\right)$. symmetries. The antiferromagnetic state therefore allows for a linear ME effect given by the ME tensor with only one independent non-zero component $\chi_{a a}=-\chi_{b b}$ (ref. ${ }^{31}$ ), or equivalently $\chi_{X Y}=\chi_{Y X}$, which was experimentally verified ${ }^{30}$. Notably, the ME tensor of this form already supports the existence of ferroic order of pure $q_{a^{2}-b^{2}}$ quadrupoles 7,8 . In addition, the existence of this quadrupole order is also understandable by a close look at the spin arrangement. The four $\mathrm{Cu}^{2+}$ spins within each $\mathrm{Cu}_{4} \mathrm{O}_{12}$ cluster are roughly perpendicular to the approximately flat $\mathrm{CuO}_{4}$ plane, whose $X Y$-plane components are combined into a cluster $q_{a^{2}-b^{2}}$ quadrupole moment. Moreover, the $q_{a^{2}-b^{2}}$ components on the respective clusters align uniformly both in the $X Y$ plane and along the $Z$ axis, thus forming a ferroic $q_{a^{2}-b^{2}}$ type quadrupole order.

The magnetic symmetry reduction from $4221^{\prime}$ to $4^{\prime} 22^{\prime}$ upon the ordering produces a pair of quadrupole domains, labeled as Q1 and Q2, which are mutually converted by either fourfoldrotation or time-reversal operations, as drawn in Fig. $2 b, c$. These domains have opposite signs of the ME coefficient $\chi_{a a}$ to each other and hence of the ME energy $F_{\mathrm{ME}}=-\chi_{a a} E_{a} B_{a}$. A monodomain state of Q1 or Q2 is therefore achieved by the simultaneous application of poling $E_{a}$ and $B_{a}$ during the cooling process, referred to as $E_{a}^{p}$ and $B_{a}^{p}$, respectively. Figure $2 \mathrm{~d}$ shows the $B_{a}$ dependence of electric polarization along the $a$ axis $\left(P_{a}\right)$ after cooling with four different combinations of $E_{a}^{p}$ and $B_{a}^{p}$, where the slope corresponds to $\chi_{a a}$. In accordance with the expected domain selection by the ME cooling procedure, $\chi_{a a}>0$ for the two parallel configurations $\left(+E_{a}^{p},+B_{a}^{p}\right)$ and $\left(-E_{a}^{p},-B_{a}^{p}\right)$, while $\chi_{a a}<0$ for the two antiparallel configurations $\left(+E_{a}^{p},-B_{a}^{p}\right)$ and $\left(-E_{a}^{p}\right.$, $\left.+B_{a}^{p}\right)$. The value of $\left|\chi_{a}\right|$ obtained from these data is $\sim 8.9 \mathrm{ps} \mathrm{m}^{-1}$, which is roughly comparable to those of typical linear $\mathrm{ME}$ materials such as $\mathrm{Cr}_{2} \mathrm{O}_{3}\left[\chi_{a a}=0.73 \mathrm{ps} \mathrm{m}^{-1}\right.$ and $\chi_{c c}=4.14 \mathrm{ps} \mathrm{m}^{-1}$ (ref. $\left.\left.{ }^{32}\right)\right]$ and $\mathrm{GaFeO}_{3}\left[\chi_{b c}=21 \mathrm{ps} \mathrm{m}^{-1}\right.$ (ref. ${ }^{33}$ )]. While the sign of $\chi_{a a}$ in each domain has not been determined experimentally, we assume $\chi_{a a}>0$ for Q1 and $\chi_{a a}<0$ for Q2 for convenience. With this assumption, Q1 and Q2 are selected in parallel and antiparallel configurations of $E_{a}^{p}$ and $B_{a}^{p}$, respectively.

Predicted optical magnetoelectric effect. Before presenting experimental results, we discuss the OME effect predicted in $\mathrm{PbTCPO}$, for light propagating along the $Z$ axis, i.e., $\mathbf{k}=\left(0,0, k_{Z}\right)$. Because of the tetragonal symmetry (magnetic point group $4^{\prime} 22^{\prime}$ ), non-ME contributions from dielectric permittivity yield an isotropic optical response for light polarized within the $X Y$ plane $^{31}$. By contrast, the OME effect defined by $\chi_{X Y}^{\omega}=\chi_{Y X}^{\omega}$ causes an anisotropy for the $X$ and $Y$ axes. This is because electric polarization induced magnetoelectrically by a magnetic field of light, $\mathbf{P}_{\mathrm{ME}}^{\omega}=\left(P_{X}^{\omega}, P_{Y}^{\omega}, P_{Z}^{\omega}\right)$, is given by ${ }^{16,17}$

$$
\begin{aligned}
\mathbf{P}_{\mathrm{ME}}^{\omega} & =\chi^{\omega} \mathbf{B}^{\omega}=\left(1 / \tilde{\mu}^{\omega} \omega\right) \chi^{\omega} \mathbf{k} \times \mathbf{E}^{\omega} \\
& =\left(k_{Z} / \mu_{X X}^{\omega} \omega\right)\left(\begin{array}{ccc}
\chi_{X Y}^{\omega} & 0 & 0 \\
0 & -\chi_{X Y}^{\omega} & 0 \\
0 & 0 & 0
\end{array}\right) \mathbf{E}^{\omega},
\end{aligned}
$$

where $\mu_{X X}^{\omega}\left(=\mu_{Y Y}^{\omega}\right)$ is the component of the magnetic permeability $\tilde{\mu}^{\omega}$. The anisotropy is reversed by switching the light propagation direction $\left(+k_{Z} \leftrightarrow-k_{Z}\right)$. Consequently, nonreciprocal linear dichroism (compare Fig. 1a, c) and birefringence show up as a manifestation of the OME effect in the ferroic quadrupole ordered state of PbTCPO. This magnetoelectrically induced optical anisotropy in an originally isotropic medium is distinct from the conventional magnetically induced one known as the reciprocal Cotton Mouton effect, and has never been observed experimentally to date. In the present study, we utilize linear dichroism to visualize the spatial distribution of quadrupole 
domains in PbTCPO. We define linear dichroism as $\Delta A_{X Y}=\left(A_{X}\right.$ $\left.-A_{Y}\right)$ and the non-OME contribution as $A_{0}=\left(A_{X}+A_{Y}\right) / 2$, where $A_{X}$ and $A_{Y}$ represent the absorption coefficients for the light linearly polarized along the $X\left(E_{X}^{\omega}\right)$ and $Y$ directions $\left(E_{Y}^{\omega}\right)$. Because $\Delta A_{X Y}$ is opposite in sign between the Q1 and Q2 domains with $\chi_{X Y}^{\omega}$ of opposite signs, $A_{X}$ and $A_{Y}$ for $+k_{Z}$ are reformulated as

$$
\begin{aligned}
& \left.\begin{array}{l}
A_{X}=A_{0}+\Delta A_{X Y} / 2 \\
A_{Y}=A_{0}-\Delta A_{X Y} / 2
\end{array}\right\} \quad \text { for Q1 domain, } \\
& \left.\begin{array}{l}
A_{X}=A_{0}-\Delta A_{X Y} / 2 \\
A_{Y}=A_{0}+\Delta A_{X Y} / 2
\end{array}\right\} \quad \text { for Q2 domain. }
\end{aligned}
$$

These relations are schematically illustrated in Fig. $1 \mathrm{a}-\mathrm{d}$. Accordingly, either $X$ - or $Y$-polarized light can be used to distinguish Q1 and Q2 with the contrast being determined by $\Delta A_{X Y}$. No domain contrast is expected for [100]-polarized light and unpolarized light.

It should be noted that the crystal structure of PbTCPO is chiral $\left(P 42_{1} 2\right)$, so that the optical (or dynamical) ME tensor $\chi^{\omega}$ can have not only time-reversal odd components as considered in Eq. (1), but also time-reversal even components that are responsible for natural optical activity ${ }^{16,34,35}$. (The time-reversal even components vanish at a static limit, as consistent with the time-reversal odd nature of static ME tensor $\chi$.) As is well known, the natural optical activity by itself does not lead to any linear anisotropy. In addition, it is allowed to show up even in the paramagnetic phase and insensitive to the label of magnetic quadrupole domains (Q1 or O2) due to their different transformation property with respect to time reversal. Thus, the natural optical activity is insignificant for magnetic quadrupole domain imaging in PbTCPO. On the other hand, the presence of the finite natural optical activity would modify $A_{X}$ and $A_{Y}$, so that the magnitude of $\Delta A_{X Y}$ may be influenced by the natural optical activity. However, as described in Supplementary Note 1, our experimental results demonstrated later indicate that, although the contribution from the natural optical activity to $\Delta A_{X Y}$ may be finite, it is small and insignificant for the quadrupole domain imaging in $\mathrm{PbTCPO}$.

Observation of nonreciprocal linear dichroism. To explore the OME effect in the near-infrared to visible light region, suitable for imaging applications, we first identify a photon energy $\left(E_{\mathrm{ph}}\right)$ region of crystal-field $d-d$ transitions of $\mathrm{Cu}^{2+}$ ions, because such transitions are responsible for OME effects in several $3 d$ systems ${ }^{24,25}$. Figure $3 \mathrm{a}$ shows wide-range $(1.2-3.4 \mathrm{eV})$ fundamental absorption spectra for unpolarized light propagating along the $Z$ axis of the crystal, measured at $4.2,8.0$, and $290 \mathrm{~K}$ (for the experimental setup, see "Methods" 36 and Supplementary Fig. 3). An increased absorption above $3 \mathrm{eV}$ and relatively weak absorption in the 1.4-2.2 eV region are separated by a transparent gap, in agreement with the blue color of a crystal (see the inset of Fig. 3a). On cooling down to $8.0 \mathrm{~K}\left(>T_{\mathrm{N}}\right)$, peak structures in the $1.4-2.2 \mathrm{eV}$ region become more evident and at least three broad peaks, located at $1.4,1.8$, and $1.9 \mathrm{eV}$, can be distinguished. The spectrum at $4.2 \mathrm{~K}\left(<T_{\mathrm{N}}\right)$ overlaps that at $8.0 \mathrm{~K}$, which is consistent with the absence of crystal symmetry lowering upon the magnetic quadrupole ordering reported by a powder neutron diffraction study ${ }^{30}$. Considering previous optical studies on $\mathrm{Cu}^{2+}$-based oxide insulators ${ }^{37,38}$, these peaks are most likely attributable to intra-atomic $d-d$ transitions of $\mathrm{Cu}^{2+}$ ions. We therefore explore the OME effect in this $E_{\mathrm{ph}}$ region.

Absorption spectra measured at various conditions, shown in Fig. 4, provide evidence for the OME effect. Before each measurement, we performed the ME-cooling procedure with
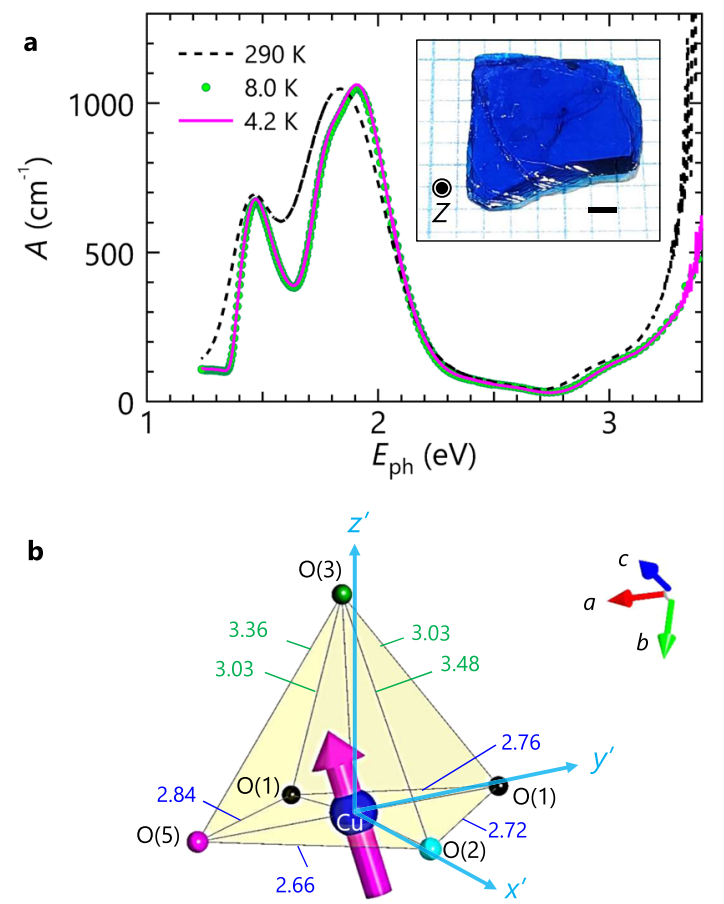

Fig. 3 Optical absorption spectra and local environment of the $\mathbf{C u}^{2+}$ ion. a Absorption spectra of a $\mathrm{Pb}(\mathrm{TiO}) \mathrm{Cu}_{4}\left(\mathrm{PO}_{4}\right)_{4}$ crystal in the photon energy range $1.2<E_{\mathrm{ph}}<3.4 \mathrm{eV}$ taken at room temperature (black dashed line), 8.0 $\mathrm{K}\left(>T_{\mathrm{N}}\right)$ (green circles), and $4.2 \mathrm{~K}\left(<T_{N}\right)$ (magenta solid line). The light is unpolarized and propagates along the $Z$ axis of the crystal. The transparent gap of 2.4-3.2 eV is consistent with the blue color of the crystal whose photo is shown in the inset (scale bar, $1 \mathrm{~mm}$ ). b Distorted $\mathrm{CuO}_{5}$ square pyramid. The site symmetry of the $\mathrm{Cu}^{2+}$ ion is 1 . The numbers denote the $\mathrm{Cu}-\mathrm{O}$ and $\mathrm{O}-\mathrm{O}$ bond distances in unit of $\AA . x^{\prime}, y^{\prime}$, and $z^{\prime}$ denote local coordinate axes.

parallel or antiparallel configuration of $E_{a}^{p}$ and $B_{a}^{p}$ to achieve a mono-domain state of either Q1 or Q2, and then switched off $E_{a}^{p}$ and $B_{a}^{p}$ to exclude any possibility of field-induced optical effects. Figure $4 \mathrm{a}$ shows absorption spectra at $4.2 \mathrm{~K}$ for $X$-polarized light $\left(A_{X}\right)$ propagating along the $+Z$ direction. Significantly, we observe a clear difference between $A_{X}$ for Q1 $\left[\left(E_{a}^{p}, E_{a}^{p}\right)=\right.$ $( \pm, \pm)]$ and Q2 $( \pm, \mp)$, as shown in the inset of Fig. 4a. Moreover, as displayed in Fig. 4b, the domain-dependent part of the absorption spectrum $[\Delta A=A(+,+)-A(-,+)]$ is completely reversed between $X$ - (black line) and $Y$-polarized light (blue line), while it vanishes for the [100]-polarized light (magenta line) and unpolarized light (green line). This feature is in perfect agreement with the linear dichroism induced by the OME effect predicted in PbTCPO (see the previous subsection and Fig. 1a-d). Furthermore, as seen in Fig. 4c, the domain-dependent part of the spectrum for the $X$-polarized light is completely reversed by switching the light propagation direction $\left(+k_{Z} \leftrightarrow-k_{Z}\right)$, which provides direct evidence of the nonreciprocity. The disappearance of the $\Delta A$ signal in the paramagnetic phase is also confirmed (see Supplementary Fig. 4). We have thus established that the ME quadrupole order in PbTCPO exhibits nonreciprocal linear dichroism induced by the OME effect (Fig. 1). Accordingly, the domain-dependent part of the absorption spectrum $\Delta A$ for the $X$ or $Y$-polarized light (black and blue lines in Fig. 4b) exactly corresponds to the nonrecirpocal linear dichroism spectra $\Delta A_{X Y}$, as indicated by Eqs. (2) and (3) (see also Fig. 1a-d).

It is noteworthy that the magnitude of the observed nonreciprocal linear dichroism in PbTCPO is quite large. The 
a

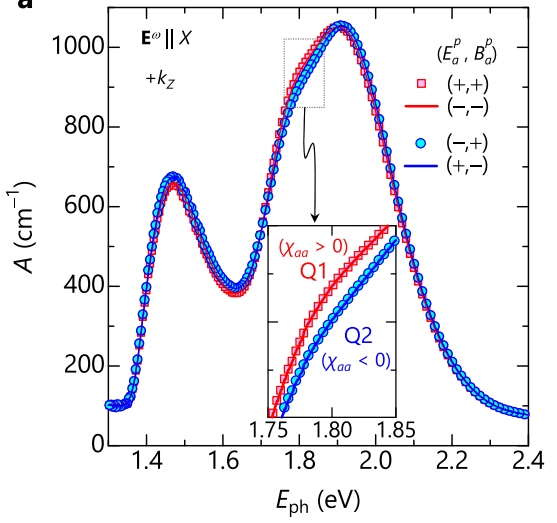

b $\quad \Delta A=A(+,+)-A(-,+)$

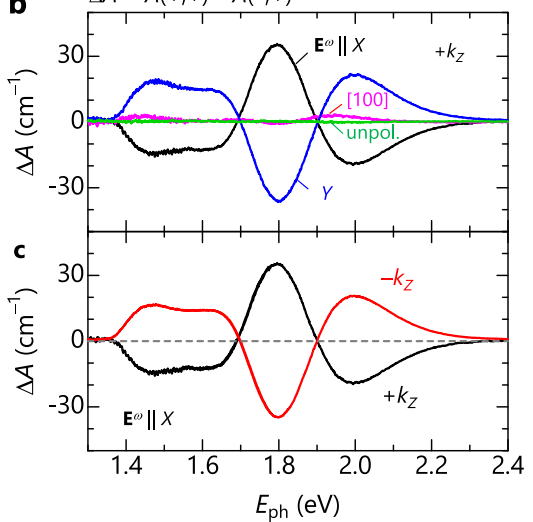

Fig. 4 Evidence for nonreciprocal linear dichroism. a Absorption coefficient $(A)$ spectra at $4.2 \mathrm{~K}$ for $X$-polarized light propagating along the $+Z$ direction, after cooling with four different sign combinations of poling fields $\left(E_{a}^{p}, B_{a}^{p}\right)$. The poling fields are removed before each measurement. The inset of a displays the magnified view in the 1.75-1.85 eV region. b Subtracted absorption spectra $\Delta A=A(+,+)-A(-,+)$ for $X-, Y-,[100]$-polarized, and unpolarized light. c $\Delta A$ spectra for $X$-polarized light propagating along the $-Z$ direction, together with that along $+Z$ for comparison.

relative difference $\Delta A_{X Y} / A_{0}$ reaches $\sim 4 \%$ at $1.8 \mathrm{eV}$, which is two orders of magnitude larger than the reported OME effects for visible light in antiferromagnetic materials [e.g., $\sim 10^{-4}$ for $\mathrm{Cr}_{2} \mathrm{O}_{3}$ (refs. ${ }^{20-22}$ )]. With such a large value of $\Delta A_{X Y} / A_{0}$, we expect that the nonreciprocal linear dichroism demonstrated here can be applied to quadrupole domain imaging, by means of simple optical methods like linear polarization microscopy.

Optical imaging of quadrupole domains. To demonstrate optical imaging of quadrupole domains, we employ a home-built linear polarization microscope with transmittance geometry and use the light wavelength of $700 \mathrm{~nm}(\approx 1.8 \mathrm{eV})$ which yields the largest $\Delta A_{X Y}$ (Fig. 4b) (for experimental details, see "Methods" and Supplementary Fig. 5). While an optical microscopy image of a $Z$-plane sample (thickness $d=50 \mu \mathrm{m}$ ) for the $X$-polarized light is uniform in the paramagnetic phase (see Supplementary Fig. 6), the contrast of the image appears at $5 \mathrm{~K}$ after zero-field cooling $\left(E_{a}^{p}=B_{a}^{p}=0\right)$, as displayed in Fig. 5a. In accordance with the light polarization dependence of the OME effect in PbTCPO (see Fig. 4b), the observed striped like contrast is reversed for the $Y$-polarized light (Fig. 5b) and disappears for the [100]-polarized and unpolarized light (Supplementary Fig. 7h, i). Note that the raw images in Fig. $5 \mathrm{a}, \mathrm{b}$ contain predominantly polarization-independent contributions that weaken the contrast. To eliminate such obstacle contributions, we generate a linear dichroism $\left(\Delta A_{X Y}\right)$ image using two images, which is obtained by calculating $\Delta A_{X Y}=-\ln \left(I_{X} / I_{Y}\right) / d$ for each pixel of the camera sensor, where $I_{X}$ and $I_{Y}$ represent the transmitted light intensity for the $X$ - and $Y$-polarized light, respectively. The resulting linear dichroism image exhibits much greater contrast, as presented in Fig. $5 \mathrm{c}$. This image ensures that the contrast is completely reversed by changing the light wavelength to $600 \mathrm{~nm}$ $(\approx 2.1 \mathrm{eV})$ (see Supplementary Fig. 7e) and $760 \mathrm{~nm}(\approx 1.6 \mathrm{eV})$ (Supplementary Fig. 7o), as well as switching the propagation direction $\left(+k_{Z} \leftrightarrow-k_{Z}\right)$ (Supplementary Fig. 7r). These wavelength and propagation direction dependences of the contrast are in excellent agreement with those of the $\Delta A_{X Y}$ spectra (see Fig. $4 \mathrm{~b}, \mathrm{c}$ ), evidencing that the contrast of the images obviously reflects the spatial distribution of the nonreciprocal linear dichroism, that is, ME quadrupole domains. We also confirm that a mono-domain state of the quadrupole order is achieved by the ME cooling procedure (see "Methods"), as indicated by the uniform linear dichroism image presented in Fig. 5e. The value of $35(2) \mathrm{cm}^{-1}$ for the Q1 state and
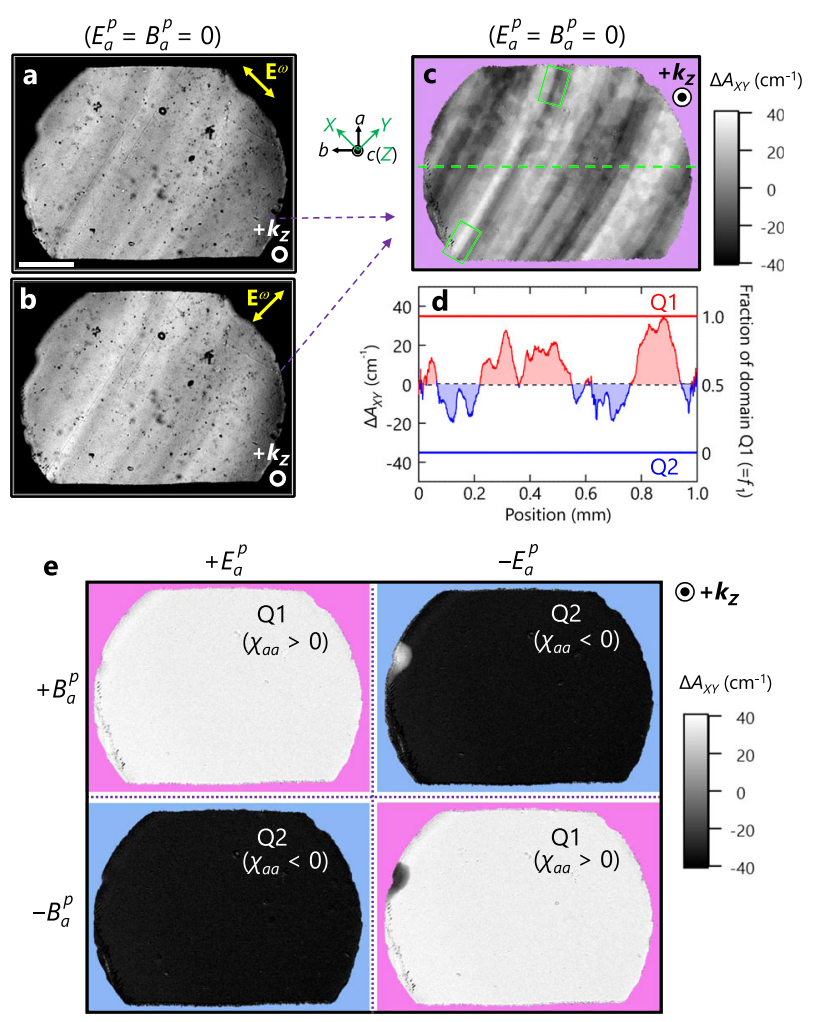

Fig. 5 Demonstration of optical imaging of magnetoelectric quadrupole domains. Microscopy images of a zero-field-cooled sample taken at $5 \mathrm{~K}$ using the $X-(\mathbf{a})$ and the $Y$ - (b) polarized light propagating along the $+Z$ direction. Scale bar, $200 \mu \mathrm{m}$. c Linear dichroism $\left(\Delta A_{X Y}\right)$ image obtained by calculating logarithmic subtraction of the image in $\mathbf{a}$ from $\mathbf{b}$ The presence of contrast indicates a multidomain state of Q1 and Q2. In the region of lower and upper green boxes, the angles between the domain boundaries and the $a$ axis are about $30^{\circ}$ and $16^{\circ}$, respectively. $\mathbf{d}$ Profile of $\Delta A_{X Y}$ (left axis) and the fraction of domain Q1, $f_{1}$, (right axis) along the green dashed line in $\mathbf{c}$. The upper red (lower blue) line denotes a single domain state of Q1 (Q2). e $\Delta A_{X Y}$ images after cooling with four different sign combinations of poling fields $\left(E_{a}^{p}= \pm 0.57 \mathrm{MV} \mathrm{m}^{-1}, B_{a}^{p}= \pm 0.1 \mathrm{~T}\right)$. The poling fields are removed before taking each image. The absence of contrast shows the single domain of Q1 (Q2) in the (anti)parallel configuration of $E_{a}^{p}$ and $B_{a}^{p}$. 

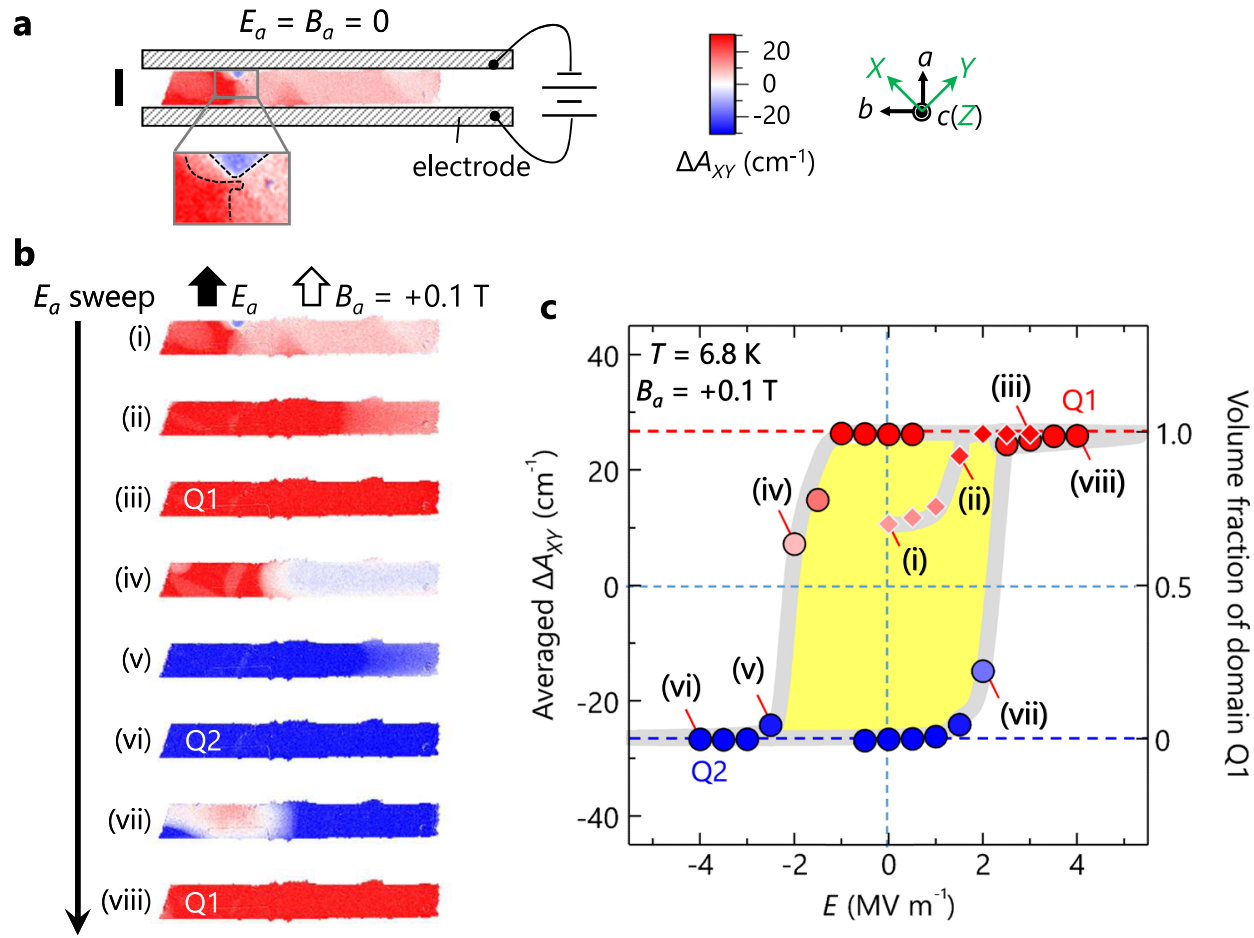

Fig. 6 Optical visualization of isothermal electric-field switching of magnetoelectric quadrupole domains. a Linear dichroism ( $\Delta A_{X Y}$ ) image of a sample with a narrow electrode gap $(100 \mu \mathrm{m})$ taken at $6.8 \mathrm{~K}$ after zero-field cooling. Scale bar, $100 \mu \mathrm{m}$. In the magnified view, domain boundaries in the region of the gray box are denoted by the black dashed lines. $\mathbf{b}$ Linear dichroism images in a sequentially applied electric field along the $a$ axis $\left(E_{a}\right)$ in the presence of a bias magnetic field along the $a$ axis $\left(B_{a}=+0.1 \mathrm{~T}\right)$. Note that more red (blue) color of the images means a larger fraction of domain Q1 (Q2). c The $E_{a}$ dependence of the linear dichroism averaged over the entire sample area (left axis) and a volume fraction of domain Q1 (right axis). The square and circle symbols represent the virgin curve and the subsequent hysteresis curve, respectively. The Roman numerals correspond to the images in $\mathbf{b}$. The hysteresis with a clear saturation confirms a complete electric-field switching of quadrupole domains.

$-35(2) \mathrm{cm}^{-1}$ for the Q2 state is quantitatively consistent with the result of the $\Delta A_{X Y}$ spectra (Fig. $4 \mathrm{~b}$ ). We therefore succeed in demonstrating the visualization of $\mathrm{ME}$ quadrupole domains in $\mathrm{PbTCPO}$ and their manipulation by the ME cooling procedure. To the best of our knowledge, this is the first demonstration of an optical imaging of antiferromagnetic domains free from net magnetization through the linear OME effect.

By comparing Fig. $5 c$ with Fig. 5e, we find that the magnitude of $\Delta A_{X Y}$ of the multi-domain state is smaller than $\pm 35(2) \mathrm{cm}^{-1}$ of the mono-domain state at most of the sample positions. Moreover, as displayed in Fig. $5 \mathrm{~d}$, the line profile of $\Delta A_{X Y}$ along the green dashed line in Fig. $5 \mathrm{c}$ does not show stepwise changes with discrete levels but rather gradually changes with changing position. Note that the spatial resolution of the microscope setup is better than $4 \mu \mathrm{m}$, so that the gradual change is not due to the instrumental resolution. These results indicate that the quadrupole domains in the zero-field-cooled sample are distributed not only in the $X Y$ plane, but also along the depth $(Z)$ direction. If we introduce a parameter $f_{1}\left(1-f_{1}\right)$ as a fraction of the Q1 (Q2) domain along the depth direction, the value of $2 f_{1}-1$ is equal to $\Delta A_{X Y}$ for the multi-domain state divided by $\Delta A_{X Y}$ for the monodomain state. Thus, we can quantitatively evaluate the domain fraction in the present sample at any position. For example, the right axis of Fig. 5d represents the fraction of the Q1 domain $\left(f_{1}\right)$ along the dashed line in Fig. $5 c$. The present domain imaging based on transmittance microscopy therefore provides quantitative information of a two dimensional distribution of the quadrupole domain fraction along the depth direction.

Isothermal electric-field switching of quadrupole domains. After establishing the quadrupole domain imaging via the OME effect, we finally turn to examine isothermal switching of quadrupole domains with electric fields. Since the ME energy is given by $-\chi_{a a} E_{a} B_{a}$ for Q1 and $+\chi_{a a} E_{a} B_{a}$ for Q2, the application of a strong positive (negative) $E_{a}$ in the presence of a positive bias $B_{a}$ can in principle switch Q2 to Q1 (Q1 to Q2). Figure 6a displays a linear dichroism image of a sample used for this switching experiment, taken at $6.8 \mathrm{~K}$ just below $T_{\mathrm{N}}$ after zero-field cooling. For unknown reasons the zero-field-cooled sample exhibits a slightly positive value of the linear dichroism and hence favors the Q1 domain. We utilize a relatively narrow electrode gap of 100 $\mu \mathrm{m}$ in order to apply a strong enough $E_{a}$. Figure $6 \mathrm{~b}$ shows a series of linear dichroism images in the switching experiment with a bias $B_{a}$ of $+0.1 \mathrm{~T}$, where we increase $E_{a}$ from 0 [panel (i)] to 3.0 $\mathrm{MV} \mathrm{m}^{-1}$ [panel (iii)] in a stepwise manner, and subsequently decrease to $-4.0 \mathrm{MV} \mathrm{m}^{-1}$ [panel (vi)] and then increase to 4.0 $\mathrm{MV} \mathrm{m}^{-1}$ [panel (viii)]. The obvious color change of the images visually demonstrates the electric-field-induced reversal of the linear dichroism and hence of the quadrupole domains. To quantify the observed domain switching, we calculate the linear dichroism averaged over the sample as well as the volume fraction of domain Q1 at each $E_{a}$, which are plotted on the left and right axes of Fig. $6 c$, respectively. Here, the volume fraction of domain Q1 is evaluated based on the averaged linear dichroism at each $E_{a}$ divided by that at $\pm 4.0 \mathrm{MV} \mathrm{m}^{-1}\left(=26.6 \mathrm{~cm}^{-1}\right)$. It is seen that after the virgin curve the volume fraction of domain Q1 exhibits a clear hysteresis loop with saturation above the coercive field of $2.5 \mathrm{MV} \mathrm{m}^{-1}$. A complete, isothermal electric-field switching of the quadrupole domains is therefore achieved with a relatively low bias magnetic field. Although the sign selection of OME effects via an electric-field cooling procedure was previously demonstrated in various systems ${ }^{20,22,28,29}$, isothermal electric- 
field switching of OME effects inside the ordered state has been rarely encountered, especially in antiferromagnetic systems. Thus, the present material can be a unique platform to investigate electric-field-driven dynamics of antiferromagnetic domains using time-resolved optical imaging.

\section{Discussion}

In the present material $\mathrm{PbTCPO}$, we observe the nonreciprocal linear dichroism induced by the OME effect. Here, we briefly discuss the microscopic origin and the spectral shape of the nonreciprocal linear dichroism. Generally, the OME effect in the near-infrared and visible light regions is understood in terms of an interference between the electric dipole (E1) and the magnetic dipole (M1) transitions through the spin-orbit coupling, and the dominant process is ascribed to crystal-field $d-d$ transitions for $3 d$ transition-metal systems $s^{22,24,25}$. As shown in Fig. 3b, the $\mathrm{Cu}^{2+}$ ion in PbTCPO is surrounded by a distorted square-pyramidal coordination of $\mathrm{O}^{2-}$ ligands. In such a crystal field, the hole ground state of $\mathrm{Cu}^{2+}$ should have a dominant $d_{x^{\prime 2}-y^{\prime 2}}$ character ${ }^{38}$. Here, $x^{\prime}, y^{\prime}$, and $z^{\prime}$ denote local coordinate axes (Fig. 3b). The OME effect associated with the $d-d$ transition of the $\mathrm{Cu}^{2+}$ hole with a $d_{x^{2}-y^{\prime 2}}$ ground state was well studied for the canted antiferromagnet $\mathrm{CuB}_{2} \mathrm{O}_{4}$ with weak spontaneous magnetization, which exhibits a gigantic nonreciprocal directional dichroism ( $\sim 300 \%)$ due to the OME effect ${ }^{25,27}$. In $\mathrm{CuB}_{2} \mathrm{O}_{4}$, the transition to the excited state $d_{x^{\prime} z^{\prime}}\left(d_{y^{\prime} z^{\prime}}\right)$ is E1 allowed, while the transition to the $d_{x^{\prime} y^{\prime}}$ is M1 allowed. These excited states are hybridized through the spin-orbit coupling due to the ordering of the $\mathrm{Cu}^{2+}$ spins whose directions lie in the $\mathrm{CuO}_{4}$ plane. The nonreciprocal dichroism is caused by a transfer of spectral weight between these two transitions through the spin-orbit coupling. Accordingly, the sign of the nonreciprocal dichroism associated with these transitions are opposite in sign to each other, which leads to the sign reversal of the spectra as experimentally observed ${ }^{25}$. Going back to the present material $\mathrm{PbTCPO}$, the $\mathrm{Cu}^{2+}$ spins are almost perpendicular to the $\mathrm{CuO}_{4}$ plane, but have a finite in-plane component (see Fig. 3b). We can thus expect that the same transition process as $\mathrm{CuB}_{2} \mathrm{O}_{4}$ is involved in the origin of the observed nonreciprocal linear dichroism. However, as shown in Fig. $4 \mathrm{~b}$, the spectrum exhibits more complicated behavior characterized by a multiple sign reversal. This suggests that all the $3 d$ states participate in the spectrum, which is reasonable because of the lowest site symmetry 1 at the $\mathrm{Cu}^{2+}$ site (Fig. 3b) as well as a nonuniform orientation of local coordinate axes for eight $\mathrm{Cu}^{2+}$ ions in the unit cell (Fig. 2a). Moreover, the significant broadening of the spectrum points to the importance of hybridization between $\mathrm{Cu}^{2+} 3 d$ orbitals and surrounding oxygen orbitals. Understanding the microscopic mechanism of the nonreciprocal linear dichroism in PbTCPO is non-trivial, but an important issue for further exploration of large OME effects.

As already mentioned, the sample shown in Fig. $5 \mathrm{c}$ displays a peculiar stripe-like domain pattern after zero-field cooling. The boundaries are, however, not straight but curved over the sample; The angles between the boundaries and the $a$ axis vary between $\sim 30^{\circ}$ (see, e.g., the lower green box of Fig. $5 \mathrm{c}$ ) and $\sim 16^{\circ}$ (the upper green box). The range of the angle coincides neither with high symmetric crystallographic axes nor an easy axis of spins (see Fig. 2a-c). Moreover, such a stripe pattern is not observed in another sample after zero-field cooling, as shown in Fig. 6a. These observations suggest that the stripe pattern seen in Fig. $5 \mathrm{c}$ is nonintrinsic. In addition, as can be seen from the comparison between Fig. $5 \mathrm{c}$ and the magnified view around the main domain boundary in Fig. 6a, the direction of the domain boundaries differs from sample to sample. No preferential direction is thus found in the present study. We also note that, for the zero-fieldcooled sample in Fig. 5c, we repeatedly observed almost the same domain pattern below $T_{\mathrm{N}}$ after heating the sample even to room temperature far above $T_{\mathrm{N}}$. A similar memory effect of the domain structure was previously reported in the ME antiferromagnet $\mathrm{Cr}_{2} \mathrm{O}_{3}$ (ref. ${ }^{14}$ ). Domains in PbTCPO are likely pinned by either defects formed during crystal growth, or other reasons such as local thermal inhomogeneity created in the sample which might depend on the sample setup. If the latter is true, then it suggests an interesting possibility of thermal control of antiferromagnetic domains $^{39}$. Future studies to elucidate its origin are important from the viewpoint of antiferromagnetic domain engineering.

In conclusion, we have investigated the OME effect for nearinfrared to visible light due to ferroic ME quadrupole order in the tetragonal antiferromagnetic material $\mathrm{Pb}(\mathrm{TiO}) \mathrm{Cu}_{4}\left(\mathrm{PO}_{4}\right)_{4}$ (PbTCPO). Our detailed experiments with various light conditions have revealed that the OME effect in PbTCPO creates an optical anisotropy in the originally isotropic $a b$ plane, which leads to a genuine nonreciprocal linear dichroism free from any crystallographic contributions. To our knowledge, PbTCPO is the first material to exhibit such a magnetoelectrically-created optical anisotropy. The nonreciprocal linear dichroism is observed in the photon energy region corresponding to the intra-atomic $d-d$ transitions of $\mathrm{Cu}^{2+}$ ions, and the relative difference of the linear dichroism reaches $\sim 4 \%$ at $1.8 \mathrm{eV}$. With such a large value of the linear dichroism, we have succeeded in visualizing the $\mathrm{ME}$ quadrupole domains and their isothermal electric-field switching by a simple transmission optical microscopy.

As mentioned above, the ME multipoles are intrinsically antiferromagnetic entities. Thus, the present demonstration of the easy optical imaging of the ME quadrupole domains and their isothermal electric-field switching is interesting in the search for ME functionalities of antiferromagnets, such as voltagecontrollable magneto-optical devices. Moreover, since the present imaging method is based on linear optics, an exposure time shorter than $1 \mathrm{~ms}$ could be straightforwardly achieved even with a commercially available high-speed camera ${ }^{40}$. Such fast imaging allows to investigate the electric-field-driven antiferromagnetic domain dynamics in a wide range of spatial and temporal scales, which is difficult to achieve by known techniques such as second harmonic generation microscopy ${ }^{14}$, magnetoelectric force microscopy ${ }^{41}$, and scanning resonant X-ray diffraction ${ }^{42,43}$. Finally, it is also intriguing to apply the OME domain imaging to exotic phases in strongly correlated systems, where some of the ME multipoles were proposed to be a primary order parameter. Examples include a pseudo-gap phase of high- $T_{c}$ cuprate superconductors. Direct visualization of ME multipole domains via the OME effect may provide solid evidence for such an exotic proposal.

After the submission of this paper, Sato et al. ${ }^{44}$ reported magnetochiral dichroism in an antiferromagnet $\mathrm{MnTiO}_{3}$, which is related to OME effects and whose magnitude reaches $10^{-3}$ in the visible light region.

\section{Methods}

Sample preparation and characterization. Single crystals of $\mathrm{Pb}(\mathrm{TiO}) \mathrm{Cu}_{4}\left(\mathrm{PO}_{4}\right)_{4}$ were grown by slow cooling of the melt ${ }^{30}$. The orientation of the crystals was determined using Laue X-ray diffraction patterns. Because of the noncentrosymmetric space group $P 42_{1} 2$, the crystal structure has a structural chirality or handedness. Our polarized light microscopy observation at room temperature revealed that most of the obtained crystals have a monochiral domain, as in the isostructural compound $\mathrm{Sr}(\mathrm{TiO}) \mathrm{Cu}_{4}\left(\mathrm{PO}_{4}\right)_{4}$ (ref. $\left.{ }^{45}\right)$. We used monochiral crystals for all measurements, although chirality, and hence optical activity, has no direct correlation with the emergence of nonreciprocal linear dichroism. Magnetization $(M)$ measurements were performed using a commercial superconducting quantum interference device magnetometer (Quantum Design MPMS3). Electric polarization along the $a$ axis $\left(P_{a}\right)$, as function of temperature and magnetic field along the $a$ axis $\left(B_{a}\right)$, 
was obtained by integrating a displacement current measured with an electrometer (Keithley 6517). To obtain a single quadrupole domain state, before each measurement of $P_{a}$, static magnetic $\left(B_{a}^{p}= \pm 1 \mathrm{~T}\right)$ and electric $\left(E_{a}^{p}= \pm 0.83 \mathrm{MV} \mathrm{m}^{-1}\right)$ fields along the $a$ axis were simultaneously applied while cooling the sample from $10 \mathrm{~K}$ (paramagnetic phase) to $3 \mathrm{~K}$, after which these poling fields were switched off. The formation of a single domain state was confirmed by a saturation of $P_{a}$ above $\left|E_{a}^{p}\right|=0.56 \mathrm{MV} \mathrm{m}^{-1}$. The crystal structures displayed in Figs. 2 and 3 were drawn by using VESTA software ${ }^{46}$.

Optical absorption measurements. Optical absorption spectra in the photon energy range of $1.2<E_{\mathrm{ph}}<3.4 \mathrm{eV}$ were measured using a fiber-based optical system $^{36}$ as schematically illustrated in Supplementary Fig. 3, developed at the High Field Laboratory for Superconducting Materials in Tohoku University. One of the crystals was cut into a thin plate with the widest faces normal to the $Z$ axis, polished down to a thickness $d=30 \mu \mathrm{m}$, and then mounted on a quartz window attached to a sample holder. Light from a halogen lamp was guided with an optical fiber to the sample, and the transmitted light was guided with a different optical fiber to a spectrometer (Horiba iHR550) equipped with a CCD camera detector (Andor iDus DV401A). The optical resolution was $0.90 \mathrm{~nm}$. The sample and optical components were installed in a cryostat equipped with a superconducting magnet that enables an application of $B_{a}^{p}$. To apply $E_{a}^{p}$, two parallel electrodes with a $500 \mu \mathrm{m}$ gap were made on the sample surface using conductive silver paste. The voltage was generated by a voltage source (Keithley 6517). All the absorption spectra displayed in Fig. 4 were taken in the absence of external fields. Before each measurement of absorption spectra, the sample was cooled from $10 \mathrm{~K}\left(>T_{\mathrm{N}}\right)$ to $4.2 \mathrm{~K}$ with a simultaneous application of $\left|E_{a}^{p}\right|=0.20 \mathrm{MV} \mathrm{m}^{-1}$ and $B_{a}^{p}= \pm 0.1 \mathrm{~T}$ to generate a single domain state, after which these poling fields were switched off. We confirmed that saturation of the nonreciprocal linear dichroism signal $\left(\Delta A_{X Y}\right)$, and hence the formation of a single domain state, was achieved after cooling with $\left|B_{a}^{p}\right|=0.1 \mathrm{~T}$ and $\left|E_{a}^{p}\right| \geq 0.20 \mathrm{MV} \mathrm{m}^{-1}$. In the measurements, four different light polarizations were used, which were generated by a wire grid polarization film (Asahi Kasei WGFTM) attached to the sample holder. When changing light polarization direction and/or switching light propagation direction, we took out the optical probe from the cryostat, reattached a polarizer film, and then reinstalled the probe.

Optical domain imaging. Domain imaging was conducted using a home-built horizontal polarized light microscope in the transmittance geometry, as schematically illustrated in Supplementary Fig. 5. A white LED (Thorlabs MWWHF2) was used as light source. For each domain imaging at a different wavelength of light, bandpass filters with a center wavelength of 600,700 , and $760 \mathrm{~nm}(\mathrm{FWHM}=10 \mathrm{~nm})$ were used. A microscopy image was taken by a CMOS camera (Thorlabs Quantalux sCMOS Camera) with an exposure time of $0.5 \mathrm{~s}$ or less. Spatial resolution of the microscope setup is better than $4 \mu \mathrm{m}$. We prepared two platelet samples with $d=50 \mu \mathrm{m}$ and $d=60 \mu \mathrm{m}$, whose images are shown in Figs. 5 and 6, respectively. The widest faces of these samples are parallel to the $Z$ plane. Each of them was glued on an oxygen-free copper plate with a hole for light transmission, which was mounted on the cold head of a helium cryostat (Oxford Instruments, MicrostatHe). A permanent magnet was used to apply $B_{a}= \pm 0.1 \mathrm{~T}$. To apply $E_{a}$, a voltage source (Keithley 6517 ) was connected to two parallel electrodes formed on the sample surface, as schematically illustrated in Fig. 6a. The gap distance of the electrodes was about $700 \mu \mathrm{m}$ $(100 \mu \mathrm{m})$ for the sample in Fig. 5 (Fig. 6).

\section{Data availability}

The data that support the findings of this study are available from the corresponding author upon reasonable request.

Received: 16 March 2020; Accepted: 9 June 2020;

Published online: 09 July 2020

\section{References}

1. Hayami, S., Yatsushiro, M., Yanagi, Y. \& Kusunose, H. Classification of atomic-scale multipoles under crystallographic point groups and application to linear response tensors. Phys. Rev. B 98, 165110 (2018).

2. Suzuki, M.-T. et al. Multipole expansion for magnetic structures: a generation scheme for a symmetry-adapted orthonormal basis set in the crystallographic point group. Phys. Rev. B 99, 174407 (2019).

3. Spaldin, N. A. \& Ramesh, R. Advances in magnetoelectric multiferroics. Nat. Mater. 18, 203-212 (2019).

4. Astrov, D. N. The magnetoelectric effect in antiferromagnetics. Sov. Phys. JETP 11, 708 (1960).

5. Schmid, H. On ferrotoroidics and electrotoroidic, magnetotoroidic and piezotoroidic effects. Ferroelectrics 252, 41-50 (2001).
6. Fiebig, M. Revival of the magnetoelectric effect. J. Phys. D. Appl. Phys. 38, R123-R152 (2005).

7. Spaldin, N. A., Fiebig, M. \& Mostovoy, M. The toroidal moment in condensed-matter physics and its relation to the magnetoelectric effect. $J$. Phys. Condens. Matter 20, 434203 (2008).

8. Kimura, K. et al. Magnetodielectric detection of magnetic quadrupole order in $\mathrm{Ba}(\mathrm{TiO}) \mathrm{Cu}_{4}\left(\mathrm{PO}_{4}\right)_{4}$ with $\mathrm{Cu}_{4} \mathrm{O}_{12}$ square cupolas. Nat. Commun. 7, 13039 (2016).

9. Němec, P., Fiebig, M., Kampfrath, T. \& Kimel, A. V. Antiferromagnetic optospintronics. Nat. Phys. 14, 229-241 (2018).

10. Cheong, S.-W., Fiebig, M., Wu, W., Chapon, L. \& Kiryukhin, V. Seeing is believing: visualization of antiferromagnetic domains. npj Quantum Mater. 5 , 3 (2020).

11. Peng, L. et al. Controlled transformation of skyrmions and antiskyrmions in a non-centrosymmetric magnet. Nat. Nanotechnol. 15, 181-186 (2020)

12. Lovesey, S. W., Khalyavin, D. D. \& Staub, U. Ferro-type order of magnetoelectric quadrupoles as an order-parameter for the pseudo-gap phase of a cuprate superconductor. J. Phys. Condens. Matter 27, 292201 (2015).

13. Fechner, M., Fierz, M. J. A., Thöle, F., Staub, U. \& Spaldin, N. A. Quasistatic magnetoelectric multipoles as order parameter for pseudogap phase in cuprate superconductors. Phys. Rev. B 93, 174419 (2016).

14. Fiebig, M., Fröhlich, D., Sluyterman v. L., G. \& Pisarev, R. V. Domain topography of antiferromagnetic $\mathrm{Cr}_{2} \mathrm{O}_{3}$ by second-harmonic generation. Appl. Phys. Lett. 66, 2906-2908 (1995).

15. Brown, W. F., Shtrikman, S. \& Treves, D. Possibility of visual observation of antiferromagnetic domains. J. Appl. Phys. 34, 1233-1234 (1963).

16. Barron, L. D. Molecular Light Scattering and Optical Activity (Cambridge University Press, 2004).

17. Arima, T. Magneto-electric optics in non-centrosymmetric ferromagnets. J. Phys. Condens. Matter 20, 434211 (2008).

18. Lovesey, S. W. \& Balcar, E. Quantum theory of natural circular, magneto-chiral and non-reciprocal linear dichroism. Phys. Scr. 81, 065703 (2010).

19. Cheong, S.-W., Talbayev, D., Kiryukhin, V. \& Saxena, A. Broken symmetries, non-reciprocity, and multiferroicity. npj Quantum Mater. 3, 19 (2018)

20. Pisarev, R. V., Krichevtsov, B. B. \& Pavlov, V. V. Optical study of the antiferromagnetic-paramagnetic phase transition in chromium oxide $\mathrm{Cr}_{2} \mathrm{O}_{3}$. Phase Transit. 37, 63-72 (1991).

21. Krichevtsov, B. B., Pavlov, V. V., Pisarev, R. V. \& Gridnev, V. N. Spontaneous non-reciprocal reflection of light from antiferromagnetic $\mathrm{Cr}_{2} \mathrm{O}_{3}$. J. Phys. Condens. Matter 5, 8233-8244 (1993).

22. Krichevtsov, B. B., Pavlov, V. V., Pisarev, R. V. \& Gridnev, V. N. Magnetoelectric spectroscopy of electronic transitions in antiferromagnetic $\mathrm{Cr}_{2} \mathrm{O}_{3}$. Phys. Rev. Lett. 76, 4628-4631 (1996).

23. Rikken, G. L. J. A., Strohm, C. \& Wyder, P. Observation of magnetoelectric directional anisotropy. Phys. Rev. Lett. 89, 133005 (2002).

24. Jung, J. H. et al. Optical magnetoelectric effect in the polar $\mathrm{GaFeO}_{3}$ ferrimagnet. Phys. Rev. Lett. 93, 037403 (2004).

25. Saito, M., Taniguchi, K. \& Arima, T. Gigantic optical magnetoelectric effect in $\mathrm{CuB}_{2} \mathrm{O}_{4}$. J. Phys. Soc. Jpn. 77, 013705 (2008).

26. Kézsmárki, I. et al. One-way transparency of four-coloured spin-wave excitations in multiferroic materials. Nat. Commun. 5, 3203 (2014).

27. Toyoda, S. et al. One-way transparency of light in multiferroic $\mathrm{CuB}_{2} \mathrm{O}_{4}$. Phys. Rev. Lett. 115, 267207 (2015)

28. Iguchi, Y., Nii, Y. \& Onose, Y. Magnetoelectrical control of nonreciprocal microwave response in a multiferroic helimagnet. Nat. Commun. 8, 15252 (2017).

29. Kocsis, V. et al. Identification of antiferromagnetic domains via the optical magnetoelectric effect. Phys. Rev. Lett. 121, 057601 (2018).

30. Kimura, $\mathrm{K}$. et al. A-cation control of magnetoelectric quadrupole order in A ( $\mathrm{TiO}) \mathrm{Cu}_{4}\left(\mathrm{PO}_{4}\right)_{4}(\mathrm{~A}=\mathrm{Ba}, \mathrm{Sr}$, and $\mathrm{Pb})$. Phys. Rev. B 97, 134418 (2018).

31. Aroyo, M. I. et al. Crystallography online: Bilbao crystallographic server. Bulg. Chem. Commun. 43, 181 (2011).

32. Wiegelmann, H., Jansen, A. G. M., Wyder, P., Rivera, J.-P. \& Schmid, H. Magnetoelectric effect of $\mathrm{Cr}_{2} \mathrm{O}_{3}$ in strong static magnetic fields. Ferroelectrics 162, 141-146 (1994).

33. Arima, T. et al. Structural and magnetoelectric properties of $\mathrm{Ga}_{2-\mathrm{x}} \mathrm{Fe}_{\mathrm{x}} \mathrm{O}_{3}$ single crystals grown by a floating-zone method. Phys. Rev. B 70, 064426 (2004).

34. Bordács, S. et al. Chirality of matter shows up via spin excitations. Nat. Phys 8, 734-738 (2012).

35. Kuzmenko, A. M. et al. Sign change of polarization rotation under time or space inversion in magnetoelectric $\mathrm{YbAl}_{3}\left(\mathrm{BO}_{3}\right)_{4}$. Phys. Rev. B 99, 224417 (2019).

36. Sawada, Y., Kimura, S., Watanabe, K. \& Nakano, M. High-field optical spectroscopy of the spin-crossover complex $\left[\mathrm{Mn}^{\mathrm{III}}(\mathrm{Taa})\right]$. J. Low Temp. Phys. 170, 424-429 (2013). 
37. Bassi, M. et al. Optical absorption of $\mathrm{CuGeO}_{3}$. Phys. Rev. B 54, R11030-R11033 (1996)

38. Pisarev, R. V., Kalashnikova, A. M., Schöps, O. \& Bezmaternykh, L. N. Electronic transitions and genuine crystal-field parameters in copper metaborate $\mathrm{CuB}_{2} \mathrm{O}_{4}$. Phys. Rev. B 84, 075160 (2011).

39. Selzer, S., Atxitia, U., Ritzmann, U., Hinzke, D. \& Nowak, U. Inertia-free thermally driven domain-wall motion in antiferromagnets. Phys. Rev. Lett. 117, 107201 (2016).

40. Katakura, I. et al. Development of high-speed polarizing imaging system for operation in high pulsed magnetic field. Rev. Sci. Instrum. 81, 043701 (2010).

41. Geng, Y. et al. Direct visualization of magnetoelectric domains. Nat. Mater. 13, 163-167 (2014).

42. Hiraoka, Y. et al. Spin-chiral domains in $\mathrm{Ba}_{0.5} \mathrm{Sr}_{1.5} \mathrm{Zn}_{2} \mathrm{Fe}_{12} \mathrm{O}_{22}$ observed by scanning resonant $\mathrm{x}$-ray microdiffraction. Phys. Rev. B 84, 064418 (2011).

43. Johnson, R. D. et al. X-ray imaging and multiferroic coupling of cycloidal magnetic domains in ferroelectric monodomain $\mathrm{BiFeO}_{3}$. Phys. Rev. Lett. 110, 217206 (2013)

44. Sato, T., Abe, N., Kimura, S., Tokunaga, Y., \& Arima, T. Magnetochiral dichroism in a collinear antiferromagnet with no magnetization. Phys. Rev. Lett. 124, 217402 (2020).

45. Kimura, K., Sera, M. \& Kimura, T. $\mathrm{A}^{2+}$ cation control of chiral domain formation in $\mathrm{A}(\mathrm{TiO}) \mathrm{Cu}_{4}\left(\mathrm{PO}_{4}\right)_{4}(\mathrm{~A}=\mathrm{Ba}, \mathrm{Sr})$. Inorg. Chem. 55, 1002-1004 (2016).

46. Momma, K. \& Izumi, F. VESTA 3 for three-dimensional visualization of crystal, volumetric and morphology data. J. Appl. Crystallogr. 44, 1272-1276 (2011).

\section{Acknowledgements}

We wish to thank P. Babkevich, H. M. Rønnow, M. Toyoda, K. Yamauchi, T. Oguchi, and T. Arima for helpful discussions. We also thank Dr. J. K. H. Fischer for correcting the English. This work was partially supported by JSPS KAKENHI Grant Numbers JP16K05449, JP17H02917, JP17H01143, JP19H05823, and JP19H01847 and by the MEXT Leading Initiative for Excellent Young Researchers (LEADER). This work was partly performed at the High Field Laboratory for Superconducting Materials, Institute for Materials Research, Tohoku University (Project Nos 18H0014 and 19H0009).

\section{Author contributions}

K.K. and T. Kimura conceived the project and coordinated experiments. K.K. and T. Katsuyoshi performed crystal growth and measurements of magnetization, electric polarization, and optical absorption spectra. K.K. constructed an experimental setup for optical domain imaging and performed the experiments. Y.S. and S.K. constructed an experimental setup for optical measurements. K.K. and T. Kimura wrote the paper.

\section{Competing interests}

The authors declare that they have no competing interests.

\section{Additional information}

Supplementary information is available for this paper at https://doi.org/10.1038/s43246020-0040-3.

Correspondence and requests for materials should be addressed to K.K.

Reprints and permission information is available at http://www.nature.com/reprints

Publisher's note Springer Nature remains neutral with regard to jurisdictional claims in published maps and institutional affiliations.

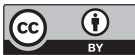

Open Access This article is licensed under a Creative Commons Attribution 4.0 International License, which permits use, sharing, adaptation, distribution and reproduction in any medium or format, as long as you give appropriate credit to the original author(s) and the source, provide a link to the Creative Commons license, and indicate if changes were made. The images or other third party material in this article are included in the article's Creative Commons license, unless indicated otherwise in a credit line to the material. If material is not included in the article's Creative Commons license and your intended use is not permitted by statutory regulation or exceeds the permitted use, you will need to obtain permission directly from the copyright holder. To view a copy of this license, visit http://creativecommons.org/ licenses/by/4.0/.

(C) The Author(s) 2020 